\title{
Déterminant possessif et accessibilité du référent dans une tâche de continuation de narrations
}

\author{
Yves Bestgen* et Sophie Piérard \\ Université catholique de Louvain, 1348 Louvain-la-Neuve, Belgique
}

\begin{abstract}
Résumé. Les facteurs qui favorisent le choix d'un type d'expressions référentielles plutôt qu'un autre par l'auteur d'un texte et ceux qui aident le lecteur à en identifier le référent ont fait l'objet de nombreuses recherches. Celles-ci ont souligné l'importance du degré d'accessibilité de l'entité en question dans le modèle mental du discours. Dans ces travaux, le fonctionnement du syntagme nominal possessif a bien peu retenu l'attention. Au moyen d'une tâche de continuation de narrations, nous avons étudié l'effet du déterminant possessif sur la mention du personnage auquel il réfère et sur la forme grammaticale employée pour y faire référence dans les suites écrites par les participants. L'impact du facteur référentiel a été comparé à celui d'un facteur connu pour affecter ce dont on parle dans un discours et comment on en parle : la présence ou non d'une rupture thématique (dans la dernière phrase fournie aux participants). Les résultats indiquent que l'emploi d'un déterminant possessif n'a pas d'effet sur la mention du "possesseur» dans les suites, mais qu'elle influence la forme grammaticale qu'il prend quand il est réintroduit, une expression anaphorique peu spécifique pouvant être employée. Le facteur thématique présente le profil inverse : la présence d'une rupture affecte la mention du possesseur dans les suites, mais non le type d'expression employée pour y faire référence. Cette conclusion est valable tant pour le français que pour l'anglais où la possession est plus marquée grammaticalement.
\end{abstract}

\begin{abstract}
Possessive determinant and accessibility of the referent in a narrative continuation task. The factors that favour the choice of one type of referential expression over another by the author of a text and those that help the reader to identify its referent have been the subject of much research. They emphasized the importance of the accessibility of the entity in question in the discourse mental model. In these studies, the role of the possessive noun phrase has received little attention. By means of a narrative continuation task, we studied the effect of the possessive determinant on the presence of the character to which it refers and on the grammatical form used to refer to it in the sentences written by the participants. The impact of this referential factor was compared to that of a factor known to affect what we say in a discourse and how we say it: the presence or absence of a thematic break (in the last sentence provided to
\end{abstract}

\footnotetext{
*Auteur-ressource : yves.bestgen@uclouvain.be
} 
the participants). The results indicate that the use of a possessive determinant has no effect on the presence of the "possessor" in the continuations, but that it influences the grammatical form used to reintroduce it, a less specific anaphoric expression being used. The thematic factor presents the opposite profile: the presence of a break affects the mention of the possessor in the continuations, but not the type of expression used to refer to it. This conclusion holds for both French and English where possession is more grammatically marked.

\section{Introduction}

Pour faire référence aux entités qui ont déjà été mentionnées dans le discours ${ }^{\mathrm{i}}$, toute une série d'expressions différentes sont disponibles: les pronoms, les noms propres, les syntagmes nominaux définis, indéfinis et démonstratifs, etc. De nombreuses recherches, tant en linguistique qu'en psychologie cognitive et en traitement automatique du langage, se sont intéressées aux facteurs qui favorisent le choix d'un type d'expression plutôt qu'un autre par le locuteur/rédacteur et à ceux qui aident le lecteur/auditeur à en identifier le référent (Clancy, 1980 ; Fox 1987 ; Grosz, Joshi \& Weinstein, 1995 ; Kehler, Kertz, Rohde \& Elman, 2008; Piérard, 2007; Vonk, Hustinx \& Simons, 1992). Parmi ceux-ci, l'accessibilité de l'entité à laquelle l'anaphore fait référence est apparue comme particulièrement importante (Ariel, 1988; Asher, Denis \& Reese, 2006 ; Chafe, 1996 ; Givón, 1983). Si l'antécédent est très accessible, une expression peu spécifique comme un pronom suffit pour y faire référence. Si l'antécédent est peu accessible, une expression plus spécifique comme la reprise du nom sera préférée. Plusieurs auteurs ont conceptualisé cette dimension sous la forme d'une échelle d'accessibilité qui attribue à chaque type d'expressions un degré d'accessibilité du référent (Ariel, 1988 ; Givón, 1983). La théorie de l'accessibilité considère que le choix d'une expression référentielle est un phénomène bidirectionnel (Ariel, 1996). L'auteur du discours la choisit en prenant en considération le degré supposé d'accessibilité de l'entité dans la représentation mentale de la personne à qui il s'adresse. Le destinataire cherche dans sa représentation mentale du discours une entité dont l'accessibilité lui est indiquée par l'expression référentielle qui est utilisée. Cette théorie soutient que, tout au long du discours, l'accessibilité des référents évolue en fonction de leur mention ou non, de l'introduction de nouveaux référents, de la présence ou non d'une rupture thématique. De nombreuses recherches ont apporté des arguments empiriques à l'appui de cette théorie (Ariel, 1996; Asher et al., 2006; Toole 1996). Toutefois, le fonctionnement dans ce cadre d'un type d'anaphore linguistiquement très spécifique a bien peu retenu l'attention : le syntagme nominal (SN) possessif comme son frère (pour faire référence au frère de Lisa).

Le SN possessif a été largement étudié en linguistique française pour ses propriétés générales, mais aussi pour ses relations avec l'anaphore associative (Bartning, 1989; Godard, 1986 ; Heinz, 2003 ; Kleiber, 2000, 2003, 2004, 2011 ; Salles, 2014, 2015). Il a pour fonction de signaler l'existence d'une relation d'appartenance (au sens large, Pattee, 1992) entre un "possesseur » et un « possédé » (Heinz, 2003). Le déterminant possessif est généralement décrit du point de vue sémantique comme la combinaison d'un article défini et d'un pronom personnel (Apothéloz, 1995 ; Kleiber, 2000), sa sæur correspondant à la sœur delà lui. Le SN possessif fait donc référence à deux entités en soulignant l'existence du lien sémantique qui les unit. Le possesseur doit être saillant alors que la tête du SN peut être employée pour introduire un nouveau référent ou pour réintroduire un référent (Salles, 2017). L'emploi d'un SN possessif devrait donc avoir plusieurs effets concurrents sur l'accessibilité des référents dans le modèle mental du discours de l'auteur comme du lecteur/auditeur. Introduire ou réintroduire le référent correspondant à la tête du SN rend évidemment celui-ci très actif, mais a aussi pour conséquence de réduire l'accessibilité des 
autres référents présents dans le modèle mental (Arnold \& Griffin, 2007). Faire référence au possesseur au moyen du déterminant possessif devrait en accroître encore le degré d'activation ou, à tout le moins, le maintenir élevé. Quelles sont les conséquences de cette double activation concurrente résultant de l'emploi d'un SN possessif sur l'évolution de l'accessibilité des référents dans le discours et donc, par exemple, sur leur mention dans la suite de celui-ci? Telle est la question au centre de la présente recherche.

Pour tenter d'y répondre, une tâche de continuation de textes a été employée (Simner \& Pickering, 2005 ; Vogels, 2014). Elle présente l'avantage de mobiliser tant les processus cognitifs à l'œuvre lors de la compréhension d'un texte, le participant devant construire un modèle mental du début de texte qui lui est fourni, que ceux à l'œuvre lors de la production puisqu'il utilise ce modèle pour produire une suite. Plus précisément, nous avons demandé à des locuteurs natifs du français d'écrire une phrase pour continuer de brèves narrations, décrivant les actions de deux personnages, dans lesquelles nous avons manipulé l'expression employée pour faire référence aux personnages. Cette expression pouvait être une anaphore possessive (son grand-père) ou la reprise du prénom (Marcel). Nous désignons dans la suite ce facteur par le terme référentiel parce que l'anaphore possessive fait référence aux deux personnages alors que le prénom ne fait référence qu'à un seul. L'intérêt d'employer des personnages comme possesseur et comme possédé est qu'il s'agit de deux entités pouvant évoluer indépendamment et que donc les participants peuvent continuer l'histoire avec chacun de ceux-ci. Les analyses ont porté sur la mention dans les suites du personnage auquel le déterminant possessif fait référence et sur le type d'expression anaphorique employé pour y faire référence, deux manières d'opérationnaliser le concept d'accessibilité du référent.

Dans cette expérience, l'impact du facteur référentiel a été comparé à celui d'un facteur connu pour affecter ce dont on parle dans un discours et comment on en parle : la continuité versus discontinuité thématique entre la dernière phrase de la narration et les phrases qui la précèdent, facteur appelé dans la suite thématique. La continuité thématique favorise la mention des entités déjà introduites dans le texte ainsi que l'emploi d'expressions anaphoriques peu spécifiques comme le pronom contrairement à une rupture thématique qui réduit l'accessibilité des référents introduits en amont (Fox, 1987 ; Piérard \& Bestgen, 2006 ; Schnedecker, 2003 ; Vonk et al., 1992). Cette comparaison nous permettra de déterminer si la différence entre les conditions déterminant possessif et prénom (facteur référentiel) est similaire à celle observée entre les conditions continuité et rupture (facteur thématique). Une réponse positive soulignerait l'importance du facteur référentiel. La seconde raison pour laquelle nous avons introduit dans l'expérience le facteur thématique est qu'il se pourrait même que le facteur référentiel ait un impact plus fort (ou même uniquement) en situation de discontinuité thématique en raison d'un possible effet plafond en situation de continuité puisque le possesseur est le personnage principal de la narration et qu'il est donc normal qu'il soit fréquemment mentionné dans les suites même s'il n'a pas été réactivé par une anaphore possessive.

La première expérience rapportée ci-dessous a été menée en français. Elle a été reproduite en anglais afin d'essayer d'établir la fiabilité des conclusions. Comparer ces deux langues présente aussi un intérêt un soi (Fabricius-Hansen, Behrens, Pitz \& Helland, 2017). En français, le choix du déterminant possessif dépend du genre de l'objet possédé et non de celui du possesseur (le frère de Lisa = son frère). En anglais, le choix du déterminant possessif dépend du genre du possesseur : his indique que le possesseur est masculin, tandis que her indique que le possesseur est féminin (the brother of Lisa=her brother). Corblin (1995) explique cette distinction en termes de différence de saturation qu'il définit de la manière suivante : " une expression est anaphorique si une part de son interprétation est une valeur non fixée, qui requiert d'être identifiée à une valeur de même type fournie par son contexte d'usage. La fixation de cette valeur sera appelée saturation » (Corblin, 1995, p. 137). Il considère qu'en anglais, tous les traits de l'anaphore possessive sont pertinents. 
Considérons deux personnages : Jack et Lisa; dans la phrase «Jack likes her car», le déterminant nous informe à propos de la personne, du nombre et du genre du possesseur. La voiture appartient à un personnage féminin ; Lisa est donc la seule candidate possible. En français, tous les traits de l'anaphore possessive ne sont pas pertinents pour la saturation. Le déterminant est accordé avec le nom de tête. Dans la phrase : «Jack aime sa voiture », la seule contrainte qui régit son antécédent est qu'il soit de la 3e personne du singulier : Jack et Lisa sont des candidats admissibles. L'identité du possesseur est donc plus marquée en anglais qu'en français. Est-ce que cette différence est suffisante pour modifier le degré d'activation de l'antécédent et donc sa mention dans les suites rédigées par les participants ? L'expérience menée en anglais vise à apporter une première réponse à cette question.

\section{Expérience 1}

\subsection{Méthode}

\subsubsection{Participants}

Soixante-treize étudiants de deuxième année en psychologie à l'Université catholique de Louvain, la très grande majorité de sexe féminin et âgée de 19 à 20 ans, ont participé à cette recherche en échange de crédits pour un cours.

\subsubsection{Matériel}

Le matériel est constitué de vingt-quatre débuts de narration de six phrases rapportant les activités de deux personnages de sexe différent dont l'un est le personnage principal et l'autre le personnage secondaire (voir le tableau 1, pour plus d'exemples, voir Piérard, 2007). Le personnage principal est introduit par son prénom en premier dans la narration et il est mentionné plus fréquemment alors que le personnage secondaire est introduit par son prénom et par un syntagme nominal possessif dont le nom de tête est un nom d'humain en relation avec un autre élément de type humain (Barque, 2015) et le déterminant possessif a pour référent le personnage principal.

Dans la dernière phrase de chaque texte, le personnage secondaire est réintroduit au moyen soit de son prénom, soit d'un syntagme nominal possessif (facteur référentiel). Le contenu de cette dernière phrase est également manipulé afin d'introduire ou non une rupture de thème (facteur thématique). Un prétest, pour lequel dix personnes ont évalué si la dernière phrase de chaque texte introduisait un événement inattendu par rapport à ce qui s'était passé avant ou si elle était une suite relativement normale à la narration en cours, a permis de confirmer l'efficacité de cette manipulation.

Tab. 1. Exemple de texte expérimental.

\begin{tabular}{|c|c|}
\hline Début du texte & $\begin{array}{l}\text { Lili va visiter le zoo avec son papy Pierrot. } \\
\text { Elle y est déjà allée l'année passée. } \\
\text { Elle court de cage en cage. } \\
\text { Pierrot lui fait faire le tour complet du zoo. } \\
\text { Lili commence à avoir très soif. }\end{array}$ \\
\hline Phrase manipulée & $\begin{array}{l}\text { Possessif et continuité } \\
\text { Son papy achète deux canettes de coca. } \\
\text { Prénom et continuité } \\
\text { Pierrot achète deux canettes de coca. } \\
\text { Possessif et rupture }\end{array}$ \\
\hline
\end{tabular}




\begin{tabular}{|l|l|}
\hline & $\begin{array}{l}\text { Son papy se rend compte qu'il a oublié sa canne. } \\
\text { Prénom et rupture } \\
\text { Pierrot se rend compte qu'il a oublié sa canne. }\end{array}$ \\
\hline
\end{tabular}

Seize textes, dits de distraction, ont également été rédigés. Ces derniers avaient pour objectif de ne pas rendre trop saillant l'objectif de l'expérience. Ces textes mentionnaient, comme les textes expérimentaux, un personnage principal et un personnage secondaire de sexe différent. Le personnage principal était réintroduit dans la dernière phrase du texte au moyen de son prénom dans la moitié des cas et d'un pronom dans l'autre moitié. Cette dernière phrase était en continuité thématique avec celles qui la précédaient.

Quatre carnets ont été constitués, chacun composé de 40 textes: les 24 textes expérimentaux et les 16 textes de distraction. Les quatre versions de chaque texte étaient réparties dans les quatre carnets de sorte qu'un même texte n'apparaissait qu'une seule fois dans chaque carnet. Chaque carnet contenait six textes expérimentaux dans chaque condition. Deux ordres de présentation des textes ont été employés. Le premier ordre a été établi de manière aléatoire en veillant toutefois à ce que la même condition expérimentale apparaisse au maximum deux fois de suite. Le deuxième ordre était l'inverse du premier.

\subsubsection{Procédure}

L'expérience se déroulait par groupes d'une dizaine de personnes. Chaque participant recevait un des quatre carnets et voyait donc toutes les conditions expérimentales (plan de type mesures répétées). Les participants avaient pour consigne de lire chaque texte et d'en écrire une suite en une phrase. L'expérimentatrice insistait sur le fait qu'ils devaient continuer et ne pas nécessairement terminer l'histoire en cours. Les participants écrivaient à leur rythme.

\subsection{Analyses et résultats}

\subsubsection{Codage des suites et procédures d'analyse statistique}

Les phrases écrites par les participants ont été codées selon qu'elles mentionnaient ou non le personnage principal. En cas de réponse positive, la forme grammaticale employée pour y faire référence était classée dans une des trois catégories suivantes : utilisation du pronom, du prénom ou d'un SN possessif. Dans la suite, seul l'emploi du pronom est analysé parce que le SN possessif est employé trop rarement pour être analysé et parce que les résultats pour le prénom sont la copie inversée de ceux pour le pronom.

Pour chaque participant et chaque condition expérimentale, on a calculé le pourcentage d'emploi du personnage principal et le pourcentage d'utilisation du pronom en employant comme numérateur la fréquence d'emploi de ce personnage. Ces deux variables dépendantes ont été analysées au moyen d'analyses de la variance pour mesures répétées à deux facteurs intraparticipants: la continuité thématique et l'expression référentielle employée dans la phrase manipulée.

\subsubsection{Mention du personnage principal}

Les pourcentages moyens de mention du personnage principal sont donnés dans le tableau 2. On y observe que celui-ci est mentionné plus souvent lorsque la phrase manipulée est thématiquement continue que lorsqu'elle introduit une rupture. L'analyse de variance confirme la présence d'un effet du facteur thématique $(F(1,72)=9,88 ; p=0,002)$. Il n'y a pas d'effet du facteur référentiel $(\mathrm{p}>0,10)$, mais bien une interaction, tout juste 
statistiquement significative au seuil de 0,05 , entre ces deux facteurs $(\mathrm{F}(1,72)=4,08$; $\mathrm{p}=0,047)$. En situation de rupture thématique, le pourcentage d'emploi du personnage principal est nettement plus grand lorsque le personnage secondaire a été réintroduit par son prénom que par un SN possessif. En situation de continuité, la différence est nettement plus faible et va même dans le sens inverse. Il apparaît donc que le déterminant possessif ne favorise pas l'emploi du possesseur dans la suite et pourrait même avoir tendance à l'inhiber lorsque le personnage secondaire est réintroduit dans une phrase en situation de rupture thématique.

Tab. 2. Mention du personnage principal dans les quatre conditions expérimentales.

\begin{tabular}{|c|c|c|}
\hline & Continuité & Rupture \\
\hline SN possessif & $77,4 \%$ & $66,5 \%$ \\
\hline Prénom & $74,7 \%$ & $72,0 \%$ \\
\hline
\end{tabular}

\subsubsection{Emploi du pronom}

Le tableau 3 présente le pourcentage moyen d'emploi du pronom pour faire référence au personnage principal dans les quatre conditions expérimentales. On observe un effet statistiquement très significatif du facteur référentiel $(F(1,71)=38,01 ; p<0,0001)$ : le personnage principal est plus fréquemment mentionné par un pronom lorsque le personnage secondaire l'a été par une anaphore possessive que lorsqu'il l'a été par son prénom. Ce résultat suggère que le déterminant possessif permet de garder le personnage principal très accessible malgré la réintroduction du personnage secondaire ; une expression référentielle moins précise peut donc être employée dans la suite pour y faire référence. L'analyse de variance ne met en évidence aucun effet du facteur thématique $(F<1)$, ni d'interaction entre les deux facteurs $(\mathrm{F}<1)$. L'expression grammaticale utilisée par les participants pour mentionner le personnage principal ne dépend donc pas de la nature thématiquement continue ou discontinue de la phrase manipulée.

Tab. 3. Emploi du pronom dans les quatre conditions expérimentales.

\begin{tabular}{|c|c|c|}
\hline & Continuité & Rupture \\
\hline SN possessif & $20,5 \%$ & $20,4 \%$ \\
\hline Prénom & $10,8 \%$ & $9,7 \%$ \\
\hline
\end{tabular}

Il est à noter que l'emploi du pronom pour faire référence au personnage principal n'est pas très fréquent dans les phrases rédigées par les participants, probablement parce qu'il n'a pas été mentionné dans la phrase manipulée qui précède celle qui doit être rédigée et que le personnage secondaire y a été réintroduit. Même si le pronom n'est pas ambigu en raison du sexe différent des deux personnages, l'emploi du prénom est préféré par les participants comme l'a déjà montré Arnold et Griffin (2007).

\subsection{Discussion}


L'expérience met en évidence des effets de chacun des deux facteurs manipulés correspondant à nos hypothèses, mais sur des variables dépendantes différentes. Le facteur thématique influence la probabilité que le personnage principal soit mentionné dans les suites alors que le facteur référentiel influence la probabilité que le pronom soit employé pour faire référence à ce personnage principal. Le premier facteur influence donc le «qui » et le second le «comment». On observe aussi une interaction entre les deux facteurs manipulés qui est dans le sens opposé à nos hypothèses. Il semble en effet que l'emploi d'un SN possessif pour réintroduire le personnage secondaire dans la phrase manipulée a tendance à réduire la fréquence de mention du personnage principal dans la phrase rédigée par les participants. Comme cette interaction est tout juste statistiquement significative, il est plus que souhaitable de déterminer si elle peut être reproduite afin d'en déterminer la fiabilité. Tel est un des objectifs de la seconde expérience. Celle-ci a été menée en anglais afin d'accroître le degré de généralité des conclusions et d'évaluer l'impact potentiel d'une différence dans la saturation anaphorique du déterminant possessif en français et en anglais. En français (et dans d'autres langues), les traits de genre et de nombre du déterminant possessif sont donnés par le nom tête du SN alors qu'en anglais (et dans d'autres langues) ils dépendent du possesseur. On peut donc penser qu'en anglais l'antécédent de l'anaphore possessive est potentiellement plus facilement identifiable et que le lien entre l'antécédent et l'anaphore est plus fort. Ceci pourrait accroître dans cette langue l'activation de l'antécédent et donc sa mention dans les suites rédigées par les participants.

\section{Expérience 2}

\subsection{Méthode}

\subsubsection{Participants, matériel et procédure}

Soixante-sept étudiants en dernière année du secondaire dans une école anglophone de Bruxelles ont participé à cette expérience. La majorité avait entre 17 et 19 ans. Les données de neuf d'entre eux n'ont pas été prises en compte, soit parce que ces participants n'étaient pas de langue maternelle anglaise, soit parce qu'ils n'avaient pas suivi les consignes. Les données de cinquante-huit participants ont donc été analysées.

Le matériel employé est identique à celui utilisé lors de l'expérience 1 à l'exception de ce qu'il a été traduit en anglais par une traductrice professionnelle et que les prénoms ont été changés afin d'être d'emploi plus fréquent en anglais (voir le tableau 4, pour plus d'exemples, voir Piérard, 2007). Le déroulement de cette seconde expérience est aussi très similaire à celui de l'expérience 1 . La seule modification notable est que l'expérience se déroulait en une seule séance, dans une grande salle.

Tab. 4. Exemple de texte expérimental en anglais.

\begin{tabular}{|c|l|}
\hline \multirow{5}{*}{ Début du texte } & Lily is visiting the zoo with her granddad, Peter. \\
& She already went last year. \\
& She rushes from cage to cage. \\
& Peter takes her all around the zoo. \\
& Lily starts to be really thirsty. \\
\hline \multirow{5}{*}{ Phrase manipulée } & Possessif et continuité \\
& Her granddad buys two cans of Coke. \\
& Prénom et continuité \\
& Peter buys two cans of Coke. \\
Possessif et rupture
\end{tabular}




\begin{tabular}{|l|l|}
\hline & $\begin{array}{c}\text { Her granddad realizes he's forgotten his walking stick. } \\
\text { Prénom et rupture } \\
\text { Peter realizes he's forgotten his walking stick. }\end{array}$ \\
\hline
\end{tabular}

\subsection{Analyses et résultats}

Les données recueillies ont été analysées comme lors de la première expérience.

\subsubsection{Mention du personnage principal}

Les pourcentages moyens de mention du personnage principal sont donnés dans le tableau 5. Comme lors de la première expérience, on y observe que le personnage principal est mentionné plus souvent lorsque la phrase manipulée est thématiquement continue que lorsqu'elle introduit une rupture $(\mathrm{F}(1,57)=9.12, \mathrm{p}=0,004)$. Il n'y a pas d'effet du facteur référentiel, ni d'interaction $(\mathrm{p}>0,10)$. On remarque toutefois que les moyennes présentent le profil inverse de celui observé lors de la première expérience, profil qui est nettement plus en accord avec les hypothèses. En situation de rupture thématique, le pourcentage d'emploi du personnage principal est plus grand lorsque le personnage secondaire a été réintroduit par un SN possessif que par son prénom. Étant donné que l'interaction n'est pas statistiquement significative, il ne serait pas correct de tirer argument de ce profil des moyennes. Celui-ci laisse néanmoins penser que l'interaction observée lors de la première expérience n'est probablement pas fiable.

Tab. 5. Mention du personnage principal dans les quatre conditions expérimentales.

\begin{tabular}{|c|c|c|}
\hline & Continuité & Rupture \\
\hline SN possessif & $68,5 \%$ & $64,4 \%$ \\
\hline Prénom & $70,0 \%$ & $59,6 \%$ \\
\hline
\end{tabular}

\subsubsection{Emploi du pronom}

L'analyse de variance met en évidence un effet statistiquement très significatif du facteur référentiel $(\mathrm{F}(1,56)=27,57, \mathrm{p}<0,0001)$. Comme lors de la première expérience, le personnage principal est plus souvent mentionné par un pronom lorsque le personnage secondaire a été réintroduit au moyen d'un SN possessif (voir le tableau 6). Il n'y a pas d'effet du facteur thématique, ni d'interaction $(p>0,10)$. Il est intéressant de constater que les pourcentages d'emploi du pronom dans les quatre conditions sont très similaires à ceux observés lors de la première expérience.

Tab. 6. Emploi du pronom dans les quatre conditions expérimentales.

\begin{tabular}{|c|c|c|}
\hline & Continuité & Rupture \\
\hline SN possessif & $24,4 \%$ & $23,3 \%$ \\
\hline Prénom & $14,3 \%$ & $10,1 \%$ \\
\hline
\end{tabular}




\section{Discussion et conclusion}

Nos expériences, tant en anglais qu'en français, mettent en évidence l'impact de l'anaphore possessive sur l'accessibilité du possesseur. Si l'anaphore possessive n'a pas d'effet sur la mention du possesseur dans les suites, elle influence par contre la forme grammaticale qu'il prend quand il est réintroduit, à savoir celle d'un pronom. Le SN possessif active donc deux entités dans la représentation mentale du lecteur. On peut supposer que le fait d'utiliser une anaphore possessive fait glisser l'attention vers le "possédé », mais le «possesseur » reste présent en arrière-plan. Cette position de second plan (et non de retrait complet) ne lui permet pas de revenir au-devant de la scène et d'être plus fréquemment réintroduit dans les suites de textes. Cependant, elle permet de le garder en position de veille et autorise l'emploi d'un pronom pour y faire référence.

Les deux expériences rapportées ci-dessus confirment donc l'impact de l'anaphore possessive sur le type d'expression référentielle employée. Par contre, nous n'observons pas d'effet de ce facteur sur la mention du personnage principal. Le deuxième facteur manipulé présente le profil inverse : l'insertion d'une rupture thématique affecte la mention de ce personnage dans les suites, mais non le type d'expression employée pour y faire référence. Si on pouvait a priori imaginer qu'un seul des deux facteurs aurait un impact ou que seul la mention ou le type d'expression référentielle serait affecté, cette dissociation n'était pas attendue. Il est toutefois possible de la présenter sous une forme nettement plus interprétable. Il apparaît en effet que la manipulation du contenu de la narration par l'introduction d'une rupture thématique affecte le contenu des suites (la mention du personnage) alors que la manipulation de la manière dont l'histoire est rédigée, par l'usage ou non d'une anaphore possessive, affecte la manière dont les participants formulent les suites (l'emploi d'un pronom). Cette opposition prend tout son sens lorsqu'elle est insérée dans le «modèle » de la production du langage de Levelt (1989; Bettoni \& Di Biase, 2015) qui soutient que les décisions à propos de ce qui sera énoncé sont prises lors de l'étape de macroplanification, une étape qui requiert d'importantes ressources cognitives et toute l'attention du locuteur, alors que la microplanification et la formulation, qui transforment les intentions communicationnelles en mots, sont des processus nettement plus automatiques. On doit toutefois garder à l'esprit que des résultats différents auraient peutêtre été observés si des ruptures thématiques plus fortes avaient été employées. Le fait que celles qui ont été utilisées affectent la mention du personnage principal indique cependant qu'elles ont un impact non négligeable sur le modèle du discours des participants à l'étude.

La comparaison des deux expériences souligne la très grande similarité des résultats obtenus en français et en anglais. Il apparaît donc que même en français, où l'accord du déterminant ne tient pas compte du genre du possesseur, celui-ci reste suffisamment accessible pour affecter la manière dont les suites sont rédigées. Si, en anglais, l'antécédent de l'anaphore possessive est potentiellement plus facilement identifiable, cela ne suffit pas à augmenter d'une manière importante l'activation de l'antécédent dans le modèle mental du discours. Il faut cependant se rappeler que, dans le matériel expérimental, l'identification de l'antécédent de l'anaphore possessive n'était en aucune manière problématique puisque la narration ne mentionnait que deux personnages et que donc le "possesseur » ne pouvait être que celui qui n'était pas « possédé ».

Une autre caractéristique du matériel expérimental mériterait une analyse approfondie afin d'en déterminer l'impact sur les résultats. Comme on peut le voir dans le tableau 1, le personnage secondaire est introduit dans les narrations au moyen de son prénom et d'un SN possessif avant que la manipulation référentielle ne soit appliquée à la sixième phrase. On peut se demander si l'impact du déterminant possessif ne serait pas plus important si ce déterminant apparaissait pour la première fois dans la phrase manipulée. Cela aurait pour conséquence qu'il ne serait plus possible de comparer le déterminant possessif au prénom puisque celui-ci ne précise pas la relation entre les deux personnages. Il serait par contre 
possible de comparer le déterminant possessif (son frère) au SN avec complément de nom (le frère de Paul). Cela présenterait le grand intérêt de permettre l'évaluation de la thèse de Kleiber selon laquelle même si l'antécédent du déterminant possessif participe à l'établissement de la référence du SN, il «n'exerce plus la même influence que dans le SN avec complément de nom, dans lequel il figure explicitement et dont il contrôle directement la référence » (Kleiber, 2000, p. 63).

Il serait aussi intéressant de compléter l'approche expérimentale employée ici par une analyse en corpus de la relation entre la présence d'un SN possessif ou d'autres expressions référentielles et la mention de l'antécédent dans les phrases qui suivent ainsi que l'expression référentielle employée. Pour que cette étude soit vraiment comparable à celle rapportée ici, il sera nécessaire de pouvoir établir de manière fiable la présence d'une rupture thématique dans le passage analysé, une tâche non triviale (Bestgen \& Piérard, 2006 ; Piérard \& Bestgen, 2006), mais qui pourrait être confiée à des annotateurs si leur degré d'accord se révèle suffisant (Artstein \& Poesio, 2008).

Yves Bestgen est chercheur qualifié du Fonds de la recherche scientifique (F.R.S-FNRS).

\section{Références bibliographiques}

Apothéloz, D. (1995). Rôle et fonctionnement de l'anaphore dans la dynamique textuelle. Genève : Droz.

Ariel, M. (1988). Referring and accessibility. Journal of Linguistics, 24, 65-87.

Ariel, M. (1996). Referring expressions and the +/- coreference distinction. In J.Gundel and T. Fretheim (eds). Referent and Referent accessibility. John Benjamins, 13-35.

Arnold, J., Griffin, Z. (2007). The effect of additional characters on choice of referring expression: everyone counts. Journal of Memory and Language, 56, 521-536.

Artstein, R., Poesio. M. (2008). Inter-coder agreement for computational linguistics. Computational Linguistics, 34, 555-596.

Asher, N., Denis, P., Reese, B. (2006). Names and pops and discourse structure. Proceedings of the workshop on Constraints in Discourse, 11-18.

Barque, L. (2015). Les noms relationnels de type humain, Langue française, 185, 29-41.

Bartning, I. (1989). Le déterminant possessif et les compléments adnominaux en de, Revue romane, $24,163-203$

Bettoni, C., Di Biase, B. (2015). Processability Theory: Theoretical bases and universal schedules, EUROSLA Monographs Series, 3, Grammatical development in second languages, 19-80.

Bestgen, Y. \& Piérard, S. (2006). Comment évaluer les algorithmes de segmentation automatique? Essai de construction d'un matériel de référence. In Actes de TALN 2006 : Verbum ex machina (pp.407-414), Presse universitaire de Louvain, Louvain-la-Neuve.

Chafe, W. (1996). Inferring identifiability and accessibility. In T. Fretheim \& J.K. Gundel (Eds). Reference and referent accessibility. Amsterdam/Philadelphia: John Benjamins.

Charolles, M. (2002). La référence et les expressions référentielles en français. Paris : Ophrys.

Clancy, P.M. (1980). Referential choice in English and Japanese narrative discourse. In W.L. Chafe (Ed.), The pear stories: cognitive, cultural, and linguistic aspects of narrative production, Vol. 3 : Advances in discourse processes. Norwood, N.J.: Ablex.

Corblin, F. (1995). Les formes de reprises dans le discours. Anaphores et chaînes de référence. Rennes : Presses universitaires de Rennes.

Fabricius-Hansen, C., Behrens, B., Pitz, A., Helland, H. P. (2017). Possessives in L2 and translation: basic principles and empirical findings, Oslo Studies in Language, 9, 3-39. 
Fox, B.A. (1987). Anaphora in popular written English narratives. In R.S. Tomlin (Ed.), Coherence and grounding in discourse. Amsterdam: Benjamins.

Givón, T. (1983). Topic continuity in discourse: quantified cross-language studies, Amsterdam: J. Benjamins.

Godard, D. (1986). Les déterminants possessifs et les compléments de nom. Langue française, 72, 102-122.

Grosz, B.J., Joshi, A.K., Weinstein, S. (1995). Centering: a framework for modeling the local coherence of discourse. Computational Linguistics, 21, 203-225.

Heinz, M. (2003). Le possessif en français : aspects sémantiques et pragmatiques. De Boeck: Duculot.

Kehler, A., Kertz, L., Rohde, H., Elman, J. L. (2008). Coherence and coreference revisited, Journal of Semantics, 25, 1-44.

Kleiber, G. (2000). Le possessif via l'anaphore associative. In M. L. Casal Silva, G. Conde Tarrío, J. Lago Garabatos, L. Pino Serrano et N. Rodríguez Pereira (éd.), La Lingüistica francesa en España camino del siglo XXI. Madrid : Arrecife. Vol. 1 : 55-74.

Kleiber, G. (2003). Un " puzzle » référentiel en anaphore associative. In F. I. Fonseca et A. M. Brito (éd.), Lingua portuguesa : estruturas, usos e contrastes. Porto : Centro de Linguística da Universidade do Porto, 97-110.

Kleiber, G. (2004). Défini associatif et possessif en concurrence textuelle. In J.-M. Adam, J.-B. Grize et M.A. Bouacha (Eds). Texte et discours : catégories pour l'analyse. Editions Universitaires de Dijon.

Kleiber, G. (2011). Sémantique et pragmatique du déterminant possessif, L'Information grammaticale, 129, 3-13.

Levelt, W.J.M. (1989). Speaking: From intention to articulation. Cambridge: MIT Press.

Pattee, J. (1992). L'emploi des déterminants avec les noms de parenté dans la Métamorphose de Kafka : un problème de traduction. TTR : traduction, terminologie, rédaction, 5, 141-167.

Piérard, S. (2007). Les anaphores possessives et les adverbiaux temporels comme marqueurs de la structure du discours. Thèse présentée en vue de l'obtention du titre de Docteur en Psychologie. Université catholique de Louvain.

Piérard, S., Bestgen, Y. (2006). Validation d'une méthodologie pour l'étude de deux types marqueurs de la segmentation dans un grand corpus de texte. Traitement Automatique des Langues, 47, 89110 .

Salles, M. (2014). Possessif ou défini associatif ? Les relations fonctionnelles et actancielles, Studii de lingvistică, 4, 199-215.

Salles, M. (2015). Anaphore possessive et anaphore associative : le cas des noms collectifs, Discours [En ligne], 16.

Salles, M. (2017). Quelques emplois discursifs des noms de parenté en général et de papa, maman, en particulier, Discours [En ligne], 20.

Schnedecker, C. (2003). La question du nom propre répété dans la théorie dite du centrage et ses problèmes, French Language Studies, 13, 105-134.

Simner, J., Pickering, M. J. (2005). Planning causes and consequences in discourse, Journal of Memory and Language, 52, 226-239.

Toole, J. (1996). The effect of genre on referential choice. In T. Fretheim and J.K. Gundel (Eds). Reference and referent accessibility. Amsterdam/Philadelphia : John Benjamins.

Vogels, J. (2014). Referential choices in language production: The role of accessibility. Tilburg: Tilburg center for Cognition and Communication. 
Vonk, W., Hustinx, L.G., Simons, W.H. (1992). The use of referential expressions in structuring discourse. Language and Cognitive Processes, 7, 301-333.

${ }^{\mathrm{i}}$ Ces expressions peuvent évidemment être aussi employées pour introduire une nouvelle entitée comme lors de l'emploi déictique d'un pronom (Charolles, 2002). 